\title{
La comprensión y el razonamiento en las conectivas proposicionales: los condicionales solo si y a menos que
}

\author{
Understanding and reasoning in the propositional connectives: \\ the conditionals solo si ('only if') and a menos que ('unless')
}

\author{
Juan Antonio García-Madruga \\ UNED - Madrid \\ jmadruga@psi.uned.es
}

ORCID iD: 0000-000I-8707-4405

Recibíu / Received: 3-IX-2018

Aceptáu / Accepted: I4-I-2019

RESUMEN. En el estudio de las conectivas proposicionales confluyen la lingüística, la lógica y la psicología, por lo que constituye un campo de gran interés para la ciencia cognitiva. Los enunciados condicionales son especialmente relevantes, ya que son una herramienta básica para expresar en forma precisa las posibilidades lógicas que se producen en la realidad. La teoría de los modelos mentales sostiene que la comprensión y el razonamiento a partir de los condicionales si entonces lleva a la construcción inicial de una única posibilidad explícita, mientras que en solo si y a menos que la representación inicial incluye dos posibilidades. No es de extrañar, por tanto, que solo si y a menos que se utilicen frecuentemente para la expresión de las advertencias y las amenazas. En estos contextos pragmáticos, además de la posibilidad deseable, se destaca aquella otra sobre la que se advierte o con la que se amenaza; por ejemplo, Aprobarás solo si estudias más o No aprobarás a menos que estudies más. En este trabajo presentamos algunas reflexiones teóricas sobre estas dos formulaciones condicionales estrechamente relacionadas, así como los resultados de tres estudios recientes realizados desde la perspectiva de la psicología cognitiva del razonamiento.

Palabras clave: condicionales, comprensión, razonamiento, representación.

ABSTRACT. The study of propositional connectives combines insights from linguistics, logic and psychology and it thus constitutes a field of great interest for cognitive science. Conditional assertions are especially relevant because they are a basic tool to express accurately the logical possibilities that occur in reality. The theory of mental models holds that understanding and reasoning on the basis of si entonces ('if then') conditionals leads to the initial construction of a single explicit possibility, while in solo si ('only if') and $a$ menos que ('unless') the initial representation includes two possibilities. It is not surprising, therefore, that solo si and a menos que are frequently used for the expression of warnings and threats. In these pragmatic contexts, both the desirable possibility and the alternative possibility on which one is warned or threatened are emphasized; for example, Aprobarás solo si estudias más ('you will pass only if you study more') or No aprobarás a menos que estudies ('you won't pass unless you study more'). In this paper we present some theoretical reflections on these two closely related conditional formulations, as well as the results of three recent studies from the perspective of the cognitive psychology of reasoning. 
Keywords: conditionals, comprehension, reasoning, representation.

Cómo citar / Form of CitATion: García-Madruga, Juan Antonio (2019): "La comprensión y el razonamiento en las conectivas proposicionales: los condicionales solo si y a menos que", Glosema, I, pp. 83-io6. https://doi.org/I0.178 I I/glosema. I.2019.83-106.

\section{INTRODUCCIÓN}

Z L estudio experimental de la interpretación y comprensión de las conectivas cionales (si entonces) — por parte de los nińos, jóvenes y adultos, así como el razonamiento a partir de los enunciados que las incluyen, ha sido una de las tareas a la que los psicólogos cognitivos han prestado especial atención en estos últimos cincuenta ańos, a partir principalmente de la llegada de la revolución cognitiva. Estos estudios están en el origen de la ciencia cognitiva y muestran de forma especialmente clara el rasgo principal de esta nueva disciplina: su carácter plenamente multidisciplinar al confluir necesariamente los conocimientos disciplinares de la lingüística, la lógica y la psicología.

Uno de los orígenes más claros de estos estudios está en el logicismo de la psicología del Piaget, con su reclamación de la lógica proposicional, con sus dieciséis combinaciones, como característica básica del pensamiento adolescente. En su intento de explicar las más altas capacidades humanas de razonamiento y, en particular, el pensamiento científico, la teoría de Piaget propone que, con la llegada de la adolescencia, los individuos adquieren la habilidad de razonar en forma hipotético-deductiva y, por tanto, de resolver los problemas deductivos de manera abstracta, formal, independiente del contenido de la tarea (Inhelder y Piaget 1972 [1955]). De esta manera, las llamadas operaciones formales características de la adolescencia y la edad adulta permitirían resolver los problemas lógicos que se expresan mediante las conectivas lógico-lingüísticas. En el cuadro I, presentamos las tablas de verdad de algunas de las principales conectivas lógicas, con su formulación lingüística en español. 


\begin{tabular}{|c|c|c|c|c|c|c|}
\hline & & $\mid \begin{array}{c}\text { ConjunCión } \\
y\end{array}$ & $\begin{array}{c}\text { DisY. } \\
\text { EXCL. } \\
o \\
\text { (pero no } \\
\text { ambos) }\end{array}$ & $\begin{array}{c}\text { DisY. } \\
\text { INCL. } \\
o \\
\text { (incluye } \\
\text { ambos) }\end{array}$ & $\begin{array}{c}\text { BICONDICIONAL } \\
\text { si y solo si enton- } \\
\text { ces }\end{array}$ & $\begin{array}{l}\text { CONDICIONAL } \\
\text { si entonces }\end{array}$ \\
\hline$p$ & $q$ & V & $\mathrm{F}$ & $\mathrm{V}$ & $\mathrm{V}$ & V \\
\hline$p$ & no- $q$ & $\mathrm{~F}$ & V & $\mathrm{V}$ & $\mathrm{F}$ & $\mathrm{F}$ \\
\hline no- $p$ & $q$ & $\mathrm{~F}$ & V & V & $\mathrm{F}$ & V \\
\hline no- $p$ & no- $q$ & $\mathrm{~F}$ & $\mathrm{~F}$ & $\mathrm{~F}$ & $\mathrm{~V}$ & $\mathrm{~V}$ \\
\hline
\end{tabular}

CuAdro i. Tablas de verdad de las conectivas lógicas más usuales ( $V=$ verdadero; $F=$ falso)

Los resultados de los estudios sobre el desarrollo del razonamiento proposicional muestran que los nińos comienzan pronto a entender y utilizar los enunciados conjuntivos y después los disyuntivos, así como a extraer las inferencias que implican ( $c f$. Delval y Carretero 1978). A partir de los 4-5 años, los niños utilizan y entienden adecuadamente una proposición conjuntiva como Esta tarde Carlos irá a ver a sus nietos y al cine, y son capaces de inferir que si Carlos no va a verlos la proposición es falsa. Posteriormente, los niños algo mayores entienden y utilizan adecuadamente enunciados disyuntivos como Esta tarde Carlos irá a ver a sus nietos o al cine, primero como disyunción excluyente ( $p$ o $q$, pero no ambos), y después como disyunción que incluye la posibilidad de que se den ambas situaciones. Así, a partir del hecho de que Carlos no haya ido a ver a sus nietos, son capaces de inferir que ha ido a al cine.

La comprensión y el razonamiento a partir de los enunciados condicionales es una tarea más compleja y tardía que las conjunciones, e incluso que las disyunciones. Johnson-Laird (I 999) propuso que los niños pequeños (hasta los 8-9 años) tratan los condicionales como conjunciones, los niños ligeramente mayores (hasta los I 2-I 4 ańos) los tratan como bicondicionales, y los adolescentes y adultos los tratan ya como condicionales unidireccionales. Esta propuesta ha sido corroborada por diversos autores ( $c f$. Taplin, Staudenmayer y Taddonio 1974; Barrouillet, Grosset y Lecas 2000; Moreno Ríos y García-Madruga 2002).

Los enunciados condicionales constituyen el campo más importante del razonamiento proposicional por su importancia en el pensamiento científico, así como por las dificultades que plantea a los individuos su comprensión y uso. Un enunciado condicional está formado por dos proposiciones, el antecedente (prótasis) y el consecuente (apódosis), y en él se afirma que si el antecedente es verdadero, entonces el consecuente debe serlo también. Si miramos la tabla de verdad del condi- 
cional —en lógica, la implicación material一, veremos que resulta relativamente peculiar, ya que es asimétrica: solo en una posibilidad, cuando el antecedente es verdadero y el consecuente es falso, el enunciado condicional es falso. Por el contrario, en los enunciados bicondicionales - la equivalencia lógica - la tabla de verdad es claramente simétrica: si ambas proposiciones son verdaderas o ambas falsas el bicondicional es verdadero; y si una de las dos es verdadera y la otra falsa, el enunciado resultante es falso.

A partir de los enunciados condicionales se pueden formular varias reglas de inferencia que han sido estudiadas ampliamente por los psicólogos: el Modus Ponens (MP: "Si $p$, entonces $q$ ", " $p$ ” luego “ $q$ "), el Modus Tollens (MT: "Si $p$, entonces $q$ ", "no- $q$ " luego "no- $p$ "), la Afirmación de Consecuente (AC: "Si $p$, entonces $q$ ", " $q$ " luego " $p$ ”) y la Negación de Antecedente (NA: "Si $p$, entonces $q$ ", "no- $p$ ” luego "no-q"). Las dos primeras inferencias (MP y MT) son inferencias válidas, mientras que las otras dos son erróneas o falacias. No obstante, AC y NA pueden ser también inferencias válidas si utilizamos una interpretación en términos de equivalencia o bicondicional de un enunciado "si entonces", algo que resulta muy usual en el lenguaje cotidiano. Por ejemplo, con frecuencia podemos oír que una madre dice a su hijo: Si terminas los deberes te llevo al cine, cuando en realidad probablemente está queriendo decir Si y solo si terminas los deberes te llevo al cine. A partir de esta interpretación bicondicional, las inferencias AC y NA son perfectamente válidas y todo el mundo las usa en la su vida cotidiana.

Numerosos estudios han mostrado que existe un claro patrón de desarrollo con la edad en la comprensión y utilización de las conectivas proposicionales que conduce a que algunos individuos jóvenes y adultos, con motivación y tiempo suficiente, sean capaces de resolver las tareas deductivas más difíciles de forma acorde con la lógica. No obstante, los resultados de estos estudios sobre el razonamiento proposicional muestran también que, en contra de lo propuesto por la teoría piagetiana, la mayoría de los adolescentes y adultos cometen numerosos errores en las tareas deductivas. Estos errores son debidos a diversos sesgos entre los que destacan las características superficiales de los problemas, el contenido y el contexto de la tarea y, en relación con ambos, los conocimientos y creencias de los individuos. No parece, por tanto, que la mayoría de los individuos sean capaces de resolver los problemas deductivos de manera abstracta, independiente del contenido de la tarea.

La concepción de Piaget, a pesar de las dificultades que tiene para explicar los errores y sesgos que muestran los estudios experimentales, está en el origen de una de las teorías psicológicas más importantes sobre el razonamiento: las teorías de "reglas mentales" de inferencia. Estas teorías de la "lógica mental" sostienen que el individuo posee, y aplica en la resolución de los problemas deductivos, una serie de 
reglas sintácticas - semejantes a las de la lógica formal- que configuran una especie de "lógica natural" ( $c f$. Braine y O’Brien 1998, Rips 1994). Estas reglas de la lógica natural permiten resolver los problemas conjuntivos, disyuntivos y condicionales, es decir, los problemas de la lógica proposicional. La dificultad de las tareas de razonamiento proposicional proviene del número de reglas que los individuos tienen que aplicar para resolver un problema. Las teorías de la lógica mental, si bien explican correctamente la competencia lógica mostrada por algunas personas especialmente capaces, no lo hacen de igual manera al enfrentarse a las dificultades y errores que cometen la mayoría de los individuos, así como a la influencia del contenido de los enunciados y de las propias creencias de los individuos. En último término, las teorías de reglas, al centrarse en los rasgos formales del razonamiento, explican mal los problemas derivados de la comprensión de los enunciados que realizan los individuos.

Por el contrario, la teoría de los modelos mentales (Johnson-Laird I983, 2006; Johnson-Laird y Byrne I99I, 2002) sostiene que razonamos no a partir de reglas sintácticas formales, sino mediante representaciones semánticas; esto es, a partir de las posibilidades mentales o modelos que construimos al comprender los enunciados verbales. La solución a un problema deductivo se extrae directamente de las posibilidades o modelos que construimos. Estos modelos son representaciones analógicas del significado de los enunciados, cuya construcción y manipulación se ven afectadas por las limitaciones de capacidad de la memoria operativa (MO), lo que explicaría el hecho de que los individuos con frecuencia realicen las inferencias a partir de representaciones incompletas o iniciales de los enunciados.

En el cuadro 2 podemos ver la representación inicial, en la que se incluyen modelos explícitos e implícitos, y la final, cuando han sido ya desplegados todos los modelos que propone la teoría para las diversas conectivas proposicionales. Como vemos, las disyunciones exigen la construcción inicial de al menos dos modelos, aunque estos no sean totalmente explícitos. Así, a partir de una disyunción excluyente como Ana es bióloga o historiadora, en la representación inicial los individuos construyen ya las dos posibilidades o modelos. No obstante, guiados por el principio de verdad, y con el propósito de no sobrecargar su MO, los dos primeros modelos incluyen solo aquella información que es verdadera; es decir, 'Ana es bióloga' en el primer modelo, y 'Ana es historiadora' en el segundo. En la representación final, los modelos están ya completamente desplegados, el primer modelo es ya 'Ana es bióloga y no es historiadora', y el segundo 'Ana no es bióloga y sí es historiadora'. Asimismo, la comprensión de los condicionales exige la construcción de dos posibilidades: una totalmente explícita, que coincide con la única posibilidad verdadera de la conjunción, y otra implícita — que en el cuadro 2 se representa con tres pun- 
tos sucesivos (. . . .) - , por la cual el individuo reconoce que hay más posibilidades verdaderas. La representación final de los condicionales incluye ya las tres posibilidades lógicas de la implicación material, mientras que en el caso de los bicondicionales se incluyen solamente las dos posibilidades lógicas de la equivalencia material.

La teoría de modelos sostiene que la clave en la explicación del razonamiento humano está en los procesos semánticos de construcción y manipulación de modelos. Dada la sobrecarga que crea en la MO la realización de las operaciones mentales que requieren las inferencias deductivas, en particular el proceso de despliegue de modelos, la teoría predice que cuanto mayor sea el número de modelos necesarios para alcanzar la conclusión, mayor será la dificultad de los problemas. Una predicción básica de la teoría de modelos es que las conjunciones, al requerir un solo modelo, serán más fáciles que las disyunciones, ya que estas exigen la construcción de al menos dos modelos en su representación inicial. Cabe destacar que esta predicción no la hacen las teorías de reglas, ya que, al postular la existencia de reglas específicas para la conjunción y la disyunción, el número de reglas o pasos en la derivación de la conclusión sería el mismo para ambos tipos de conectivas (García-Madruga et al. 200I).

\begin{tabular}{|c|c|c|c|}
\hline Conectiva & & $\begin{array}{l}\text { Posibilidades } \\
\text { InICIALES }\end{array}$ & $\begin{array}{c}\text { Posibilidades } \\
\text { Finales }\end{array}$ \\
\hline Conjunción & $p$ у $q$ & $p$ у $q$ & $p$ у $q$ \\
\hline $\begin{array}{l}\text { Disyunción } \\
\text { excluyente }\end{array}$ & $p \circ q$, pero no ambos & $\begin{array}{l}p(\text { y no- } q) \\
(\text { no- } p \text { y) } q\end{array}$ & $\begin{array}{l}p \text { y no- } q \\
\text { no- } p \text { y } q\end{array}$ \\
\hline $\begin{array}{l}\text { Disyunción } \\
\text { incluyente }\end{array}$ & $p \circ q$, o ambos & $\begin{array}{c}p(\mathrm{y} \text { no }-q) \\
(\text { no- } p \text { y) } q \\
p \text { y } q\end{array}$ & $\begin{array}{c}p \text { у no- } q \\
\text { no- } p \text { y } q \\
p \text { у } q\end{array}$ \\
\hline $\begin{array}{l}\text { Bicondicional } \\
\text { (Equivalencia) }\end{array}$ & Si y solo si $p$ entonces $q$ & $\begin{array}{l}p \text { у } q \\
\ldots\end{array}$ & $\begin{array}{c}p \text { у } q \\
\text { no- } p \text { y no- } q\end{array}$ \\
\hline $\begin{array}{l}\text { Condicional } \\
\text { (Implicación) }\end{array}$ & Si $p$ entonces $q$ & $\begin{array}{l}p \text { у } q \\
\cdot \cdot\end{array}$ & $\begin{array}{c}p \text { у } q \\
\text { no- } p \text { у } q \\
\text { no- } p \text { y no- } q\end{array}$ \\
\hline
\end{tabular}

CuAdro 2. Representación inicial y final propuesta por la teoría de los modelos mentales para las diversas conectivas proposicionales (los tres puntos en la representación inicial de los condicionales hacen referencia a un modelo implícito que no ha sido desplegado)

Con respecto a los condicionales, la teoría de modelos también predice su especial dificultad, que se pone de manifiesto en el hecho de que los adolescentes, e incluso algunos adultos, continúen cometiendo errores en la inferencia MT y, es- 
pecialmente, en la inferencia NA. Ambas inferencias exigen un despliegue de los modelos y darse cuenta de que en la NA la respuesta correcta de que no hay conclusión requiere la construcción del modelo "no- $p q$ ", que distingue el condicional del bicondicional. Además, la teoría de los modelos mentales sostiene que la utilización de contenidos y contextos específicos puede llevar a que se produzca una modulación semántica o pragmática del significado de los condicionales. Así, en un condicional como Si María bebe alcohol, entonces no bebe vino, el proceso de modulación semántico-pragmática lleva a que sean construidas solo dos posibilidades: 'María bebe alcohol y no bebe vino' y 'María no bebe alcohol y no bebe vino'. La tercera posibilidad teóricamente válida, 'María no bebe alcohol y bebe vino', es eliminada por el conocimiento que el razonador tiene de que el vino es una bebida alcohólica.

Otras teorías sostienen que entre el antecedente y el consecuente de un condicional se establece una suposición e implicación de tipo pragmático. Así, el condicional "si $p$ entonces $q$ " solicita al interlocutor que suponga el antecedente $p$, a partir del cual debe interpretarse el consecuente $q$. Las concepciones suposicionales sobre el condicional han llevado al desarrollo de teorías probabilísticas sobre el razonamiento condicional ( $c f$. Evans, Over y Handley 2005; Oberauer y Wilhelm 2003). El enfoque probabilístico considera que los condicionales no pueden ser interpretados en términos veritativo-funcionales, es decir, su verdad no depende de los valores de verdad de sus proposiciones componentes, antecedente y consecuente, sino que deben ser entendidos como más o menos creíbles o probables: su probabilidad se define como la probabilidad de que se dé el consecuente $q$, una vez que se da el antecedente $p$ : $\mathrm{P}(q / p)$. Existe en la actualidad en la psicología del razonamiento un debate entre las teorías suposicionales de corte probabilístico y las teorías veritativo-funcionales a partir de modelos y posibilidades ( $c f$. García Madruga, Santamaría y Moreno Ríos 2016). En este trabajo nos centraremos en la perspectiva teórica de las posibilidades y modelos, y sus aportaciones a la comprensión y el razonamiento a partir de los condicionales solo si y a menos que.

\section{LOS CONDICIONALES SOLO SI Y A MENOS QUE}

Hemos visto que la teoría de modelos propone que en una fase inicial los individuos representan en forma explícita solo aquellas posibilidades imprescindibles para dar cuenta del significado básico de una conectiva. Es decir, además del principio de verdad, siguen lo que podemos llamar el principio de economía en la representación. De esta manera, la representación de los condicionales "si $p$ entonces $q$ " incluye en forma explícita únicamente la posibilidad conjuntiva " $p$ y $q$ ". No obs- 
tante, durante la infancia los nińos son capaces de distinguir un condicional de una conjunción al darse cuenta de que el condicional incluye también otras posibilidades que pueden ser desplegadas. Al contrario que la conjunción, la disyunción excluyente implica ya en la fase inicial dos posibilidades o modelos, ya que no es posible dar cuenta del significado disyuntivo sin la existencia de al menos dos posibilidades. Las predicciones que surgen de esta concepción sobre la representación de las conectivas han sido ampliamente confirmadas ( $c f$. Johnson-Laird, Byrne y Schaeken 1992; García-Madruga et al. 2001).

Centrándonos en los condicionales, a partir de una formulación como Si Elisa fue al gimnasio entonces durmió bien la única posibilidad que la hace falsa es 'Elisa fue al gimnasio y no durmió bien'; las otras tres posibilidades son verdaderas. Uno de los resultados básicos de los estudios sobre razonamiento condicional es la diferencia entre las dos inferencias válidas MP y MT. Así, a partir del condicional anterior y del hecho de que Elisa fue al gimnasio, los estudios demuestran que la inmensa mayoría de los individuos (cerca del ıоo \%) son capaces de realizar el modus ponens e inferir que Elisa durmió bien. Esta inferencia se extrae directamente de la posibilidad inicial 'Elisa fue al gimnasio y durmió bien'. Sin embargo, a partir del hecho de que Elisa no durmió bien, el porcentaje de individuos que realizan la inferencia modus tollens de que 'Elisa no fue al gimnasio', aunque mayoritario, es claramente inferior (alrededor del $75 \%$ ). La inferencia MT exige desplegar una segunda posibilidad, 'Elisa no fue al gimnasio y no durmió bien', lo que explica su mayor dificultad. Estas dos posibilidades anteriores permiten realizar también las inferencias "afirmación de consecuente" y "negación de antecedente". Lo que hace inválidas a estas últimas inferencias AC y NA es el despliegue de la tercera posibilidad característica de las interpretaciones condicionales: 'Elisa no fue al gimnasio y durmió bien'.

Si bien los condicionales están ligados a las formulaciones clásicas si entonces, existen también otras formulaciones condicionales complejas afirmativas, como solo $s i$, y negativas, como a menos que, que son el objeto específico de este trabajo. Desde el punto de vista lingüístico, los esquemas condicionales complejos afirmativos como solo si (y también a condición de que, con tal de que y siempre que) "constituyen formulaciones de la excepción favorable para que algo suceda [...] y están argumentativamente orientados hacia q" (Montolío I 999: 3702). Así, la formulación "si $p$ entonces $q$ " podría ser reformulada como " $p$ solo si $q$ ", aunque en función de las relaciones de temporales y de necesidad podría ser más convincente la formulación "q solo si $p$ " (cf. Montolío I 999: 3704). Nosotros hemos optado por la primera formulación porque pone de manifiesto de forma más clara la orientación hacía $q$ característica de estas formulaciones. 
De esta manera, nuestro ejemplo anterior (Si Elisa fue al gimnasio entonces durmió bien) podría ser formulado como Elisa fue al gimnasio solo si durmió bien. Desde un punto de vista lógico, las mismas tres posibilidades que son verdaderas en la formulación si entonces continúan ahora siendo verdaderas: 'Elisa fue al gimnasio y durmió bien', 'Elisa no fue al gimnasio y no durmió bien' y 'Elisa no fue al gimnasio y durmió bien' (Jeffrey I98I, Byrne 2005). Asimismo, la posibilidad que hace falsos ambos condicionales es la misma: 'Elisa fue al gimnasio y no durmió bien'. Aunque desde un punto de vista lógico las formulaciones poseen las mismas condiciones de verdad, los lingüistas suelen resaltar que las interpretaciones bicondicionales son las preferidas en los enunciados solo si (Montolío 1999). Además, si comparamos ambas formulaciones podemos observar que se han producido algunos cambios evidentes: en la formulación con solo si ha desaparecido entonces y, lo que es más importante, la cláusula subordinada aparece detrás de cláusula principal y es $q$, no $p$. En realidad, semánticamente ambos enunciados son diferentes, ya que el enunciado Si Elisa fue al gimnasio entonces durmió bien está enfocado en la condición de ir al gimnasio para que Elisa duerma bien, mientras que el enunciado Elisa fue al gimnasio solo si durmió bien se centra en la condición de que Elisa duerma bien para que vaya al gimnasio.

En las últimas décadas un número importante de estudios experimentales han estado dedicados a la comprensión y el razonamiento de los enunciados solo si. El resultado más robusto es que la clásica diferencia entre las inferencias MP y MT propia de los enunciados si entonces desaparece con solo si (Evans I977). Un segundo resultado claramente establecido es que la comprensión e inferencia a partir de los enunciados con solo si se ve facilitada cuando su segundo componente (la cláusula subordinada) precede temporalmente a la cláusula principal, como en nuestro ejemplo Elisa fue al gimnasio solo si durmió bien. Con el propósito de dar cuenta de estos hechos, la teoría de modelos ha propuesto que los individuos piensan en dos posibilidades explícitas desde el inicio cuando comprenden y razonan a partir de las expresiones solo si (Johnson-Laird y Byrne 1989). Además, las dos posibilidades que los individuos tienen in mente cuando interpretan un enunciado solo si mantienen la preferencia por la cláusula subordinada $q$, al representar primero aquello que ha sucedido antes temporalmente. Las posibilidades lógicas son las mismas, pero la representación es diferente al cambiar el orden de los componentes de las mismas (véase el cuadro 3). 


\begin{tabular}{|c|c|c|}
\hline & $\begin{array}{l}\text { PosibILIDADES } \\
\text { INICIALES }\end{array}$ & $\begin{array}{c}\text { PosibilidAdes } \\
\text { COMPLETAS }\end{array}$ \\
\hline "si $p$ entonces $q "$ & $p$ у $q$ & $\begin{array}{c}p \text { y } q \\
\text { no- } p \text { y no- } q \\
\text { no- } p \text { y } q\end{array}$ \\
\hline " $p$ solo si $q "$ & $\begin{array}{c}q \text { у } p \\
\text { no- } q \text { у no- } p\end{array}$ & $\begin{array}{c}q \text { у } p \\
\text { no- } q \text { y no- } p \\
q \text { y no- } p\end{array}$ \\
\hline "si no $q$ entonces no $p "$ & no- $q$ y no- $p$ & $\begin{array}{l}\text { no- } q \text { y no- } p \\
q \text { у } p \\
q \text { у no- } p\end{array}$ \\
\hline "no $p$ a menos que $q$ " & $\begin{array}{c}\text { (no- } q \text { y) no- } p \\
q \text { y } p\end{array}$ & $\begin{array}{c}\text { no- } q \text { y no- } p \\
q \text { у } p \\
q \text { y no- } p\end{array}$ \\
\hline
\end{tabular}

CuAdro 3. Posibilidades propuestas por la teoría de los modelos mentales para la representación de los enunciados condicionales con si entonces, solo si, si no entonces y a menos que

Existen también construcciones condicionales complejas negativas como a menos que (o a no ser que), y otras de significado semejante como salvo que y excepto que. Los condicionales a menos que, desde el punto de vista lógico, son iguales a los condicionales negados si no entonces ( $c f$., por ejemplo, Quine 1972, Reichenbach 1947). El condicional Si Elisa fue al gimnasio entonces durmió bien podría ser formulado también introduciendo la negación y cambiando el orden de las proposiciones componentes, como: Si Elisa no durmió bien entonces no fue al gimnasio. A su vez, este condicional negado podría ser formulado como Elisa no va al gimnasio a menos que duerma bien. Desde un punto de vista lógico, las mismas tres posibilidades que eran verdaderas en la formulación si entonces continúan ahora siendo verdaderas (Jeffrey I98 I, Byrne 2005). No obstante, al igual que pasaba con solo si, tanto en si no entonces como en a menos que el orden de los componentes en la representación de cada posibilidad está cambiado. Ahora son 'Elisa durmió bien y fue al gimnasio', 'Elisa no durmió bien y no fue al gimnasio' y 'Elisa durmió bien y no fue al gimnasio' (v. cuadro 3). Asimismo, la posibilidad que hace falsos ambos condicionales es la misma: 'Elisa fue al gimnasio y no durmió bien'. No obstante, como pasaba con los condicionales complejos afirmativos, al contrario que las formulaciones "si $p$ entonces $q$ ", los condicionales a menos que están orientados hacia $q, y$ su significado es también claramente exceptivo, ya que ponen de manifiesto la condición excluyente de la relación condicional (Montolío 1999: 3707). 
La similitud entre solo si y a menos que y su carácter exceptivo fue ya observada por Geiss (1973), quien propuso que el significado de a menos que podría expresarse de otro modo como excepto si o en cualquier otro caso que. Siguiendo estas ideas, Fillenbaum (1976, I986) propuso que la conectiva que mejor expresa las oraciones a menos que no era si no entonces, sino solo si. Este autor partió de una idea que queremos resaltar en este trabajo y que veremos con más detalle más adelante: el conocimiento del contenido y del contexto pragmático es esencial para la comprensión de los condicionales y, en general, de todos los enunciados lógico-lingüísticos. Fillenbaum analizó el uso de los condicionales en las oraciones de disuasión, como las advertencias y amenazas, en las que el hablante pretende que el oyente deje de hacer algo, y en las oraciones en que se pretende que haga algo, como en las promesas. Fillenbaum encontró que en el contexto semántico-pragmático de las oraciones de disuasión y amenaza el intercambio entre si no entonces y a menos que resultaba bastante fácil, mientras que en el contexto de las promesas era mucho más difícil.

Las diferencias entre si no entonces y a menos que fueron también confirmadas por Wright y Hull (1986, I988) en sus estudios sobre la utilización de los condicionales para la expresión de las relaciones condición-acción. Un ejemplo de relaciones condición-acción en el contexto pragmático de las advertencias puede mostrar de forma clara las semejanzas y diferencias entre las tres formulaciones: si no entonces, a menos que y solo si. En la expresión Si no estudias más no aprobarás el examen, la perspectiva del hablante es relativamente neutra. Por el contrario, en $N o$ aprobarás el examen a menos que estudies más, el hablante pone el acento en la deseabilidad de la prótasis ('estudiar más') y en el carácter negativo de la apódosis, resaltado mediante una negación ('no aprobar'), lo que la hace más amenazante. Por su parte, la expresión Aprobarás solo si estudias más resalta también la deseabilidad de la prótasis, aunque sin enfatizar el carácter negativo del resultado alternativo. Además de confirmar las diferencias y semejanzas entre los tres enunciados, estas autoras encontraron que los enunciados a menos que eran más difíciles que los otros dos enunciados.

A partir de estas ideas, García-Madruga et al. (2002) propusieron para a menos que una representación inicial muy semejante a la de solo si, tal y como se muestra en el cuadro 3. Este mismo cuadro presenta también las posibilidades que se representan en los condicionales "si no $q$ entonces no $p$ ", que son una versión negada de la representación clásica de "si entonces".

Como vemos también en el cuadro 3 , la particularidad de a menos que se pone de manifiesto en que la negación del antecedente en la primera posibilidad está entre paréntesis: “(no $q$ y) no $p$ ”. De esta manera, queremos dar cuenta de la propuesta de Barbara Dancygier (1998; también en Dancygier y Sweet 2005, Traugott 
I997) sobre el significado de a menos que. Esta lingüista sostiene que los condicionales "no $p$ a menos que $q$ " se interpretan como si fueran dos enunciados: "no $p$ ", y " $p$ y $q$ "; el primero expresa el resultado probable (no $p$ ), y el segundo la única circunstancia en que el resultado contrario ( $p$ ) podría suceder. Volviendo a nuestro último ejemplo, No aprobarás el examen a menos que estudies más, para Dancygier su representación estaría formada por dos posibilidades: 'estudias más y aprobarás' y 'no aprobarás'.

El principal rasgo diferencial común de los enunciados complejos solo si y a menos que es que, al contrario que los enunciados convencionales si entonces, demandan la construcción de dos modelos iniciales. Estos dos modelos, además, van de " $q$ a $p$ " y, por otro lado, pueden ser incompletos. La teoría de modelos ha propuesto y confirmado que existe otro tipo de enunciados condicionales que requieren también una representación inicial de dos posibilidades: los condicionales contrafactuales o contrafácticos. La comprensión de un enunciado como Si Elisa hubiera ido al gimnasio entonces habría dormido bien requiere también una representación inicial de dos posibilidades: una la posibilidad real 'Elisa no fue al gimnasio y no durmió bien', y otra la posibilidad expresada en el enunciado, pero contraria a los hechos: 'Elisa fue al gimnasio y durmió bien' (cf. Byrne y Tasso I999, Byrne y Egan 2004) ${ }^{1}$.

En el próximo apartado presentaremos algunos de los principales resultados de tres estudios experimentales sobre al razonamiento utilizando las cuatro inferencias condicionales. Abordaremos, en primer lugar, los resultados tanto de precisión como de latencia de respuesta en un estudio sobre los cuatro enunciados condicionales si entonces, solo si, a menos que y si no entonces. Posteriormente, presentaremos algunos resultados de un estudio con diferentes contenidos en los condicionales solo si y a menos que. Finalmente, trataremos de comprobar la hipótesis de Dancygier sobre el significado de a menos que, mediante la comparación de estos enunciados condicionales con las formulaciones disyuntivas equivalentes.

\footnotetext{
${ }^{1}$ En este sentido, la representación de dos modelos iniciales en los enunciados solo si y a menos que y en los condicionales contrafactuales ha sido también puesta a prueba por nuestro equipo de investigación en sus estudios sobre la comprensión y el razonamiento con enunciados solo si y a menos que contrafácticos, como: Elisa habría ido al gimnasio solo si hubiera dormido bien, o Elisa no habria ido al gimnasio a menos que hubiera dormido bien (Egan, García-Madruga y Byrne 2009; García-Madruga et al. 2009)
} 
3. TREs ESTUdios EXPERIMENTALES SObRE El RAZONAMIENTO A PARTIR DE SOLO SI Y A MENOS QUE

El propósito de este apartado no es la descripción rigurosa y detallada de los trabajos, sino únicamente una presentación breve de las características y resultados principales de cada estudio, en relación con el análisis teórico que hemos presentado sobre la comprensión y el razonamiento condicional a partir de los enunciados solo si y a menos que. Una presentación más completa y precisa de los resultados de estos estudios puede verse en las publicaciones originales que se citan en el texto. Nuestro propósito es mostrar cómo la investigación empírica puede formar parte de los métodos básicos de la lingüística cognitiva como lo hace de la psicolingüística y la psicología del razonamiento.

\section{I. Precisión y latencias de las respuestas inferenciales con los cuatro enunciados condicionales}

En García-Madruga et al. 2008 (experimento 2) se comprobó la habilidad de 33 estudiantes universitarios en la evaluación de las cuatro inferencias condicionales, así como el tiempo que tardaron en dar su respuesta. Con el propósito de aclarar el procedimiento básico y características principales de los experimentos de razonamiento describiremos brevemente este estudio.

Cada uno de los participantes realizó la tarea experimental en un ordenador en el que se les presentaban los cuatro enunciados condicionales equivalentes desde el punto de vista lógico. El contenido léxico de los enunciados hacía referencia a la localización de letras y números en las dos caras de una tarjeta, es decir, era un contenido abstracto; por ejemplo:

Si hay una A por un lado, hay un I por el otro lado.

Hay una A por un lado solo si hay un I por el otro lado.

No hay una A por un lado, a menos que haya un I por el otro lado.

Si no hay un I por un lado, no hay una A por el otro lado.

En cada enunciado las letras y los números estaban aleatorizados, de forma que ningún participante recibió la misma combinación de letras y números dos veces. Cada enunciado condicional aparecía 4 veces unido a una inferencia MP, 4 a NA, 4 a AC y 4 a MT. A los participantes se les proporcionaban instrucciones sobre la tarea y se les decía que tenían que decidir si las conclusiones que se les presentaban eran "necesariamente verdaderas", "falsas" o no había conclusión. Después de unos ensayos de práctica uno de los enunciados condicionales aparecía en la pantalla. Los participantes tenían que leer el enunciado y presionar la barra de espacio. In- 
mediatamente aparecía la premisa categórica correspondiente a una de las cuatro inferencias:

MP: Hay una A por un lado (Conclusión: Hay un i por el otro lado)

NA: No hay una A por un lado (Conclusión: No hay un I por el otro lado)

AC: Hay un I por el otro lado (Conclusión: Hay una A por un lado)

MT: No hay un I por el otro lado (Conclusión: No hay una A por un lado)

De nuevo, los participantes tras leer la premisa categórica tenían que presionar la barra espaciadora momento en que aparecía en la pantalla la palabra CONCLUSIÓN seguida por la conclusión específica por cada inferencia condicional. Los participantes debían responder a la pregunta presionando una de tres teclas diferentes con las siguientes etiquetas: SÍ, NO y NO HAY CONCLUSIÓN. A los participantes se les instruía para que leyeran cada oración atentamente y que después respondiesen a la pregunta tan rápido como fuera posible. El ordenador registraba tanto la respuesta dada como el tiempo que tardaban en presionar todas las teclas.

Los resultados en términos de porcentajes de respuestas (precisión) y tiempos utilizados (latencias) pueden observarse en el cuadro 4. Como vemos, existen diferencias significativas entre las inferencias MP y MT en los enunciados de un solo modelo inicial si entonces y si no entonces, mientras esas diferencias disminuyen y no son significativas en el caso de los enunciados de dos modelos iniciales solo si y a menos que. Estas diferencias entre los enunciados condicionales clásicos y los enunciados de dos modelos en el porcentaje de respuestas se ven confirmadas por los resultados en las latencias: la diferencia en las latencias de MP y MT es clara y significativa para los enunciados de un solo modelo inicial, mientras que tienden a desaparecer y no son significativas en los enunciados de dos modelos. Los resultados confirman, por tanto, la propuesta de los dos modelos para solo si y a menos que, con una particularidad: la especial dificultad de las inferencias NA. Tanto los porcentajes de respuesta como las latencias son significativamente diferentes para NA respecto del resto de inferencias: los porcentajes son los más bajos y las latencias las más altas. Como vemos, los resultados confirman las propuestas de Fillenbaum (1986): los enunciados de dos modelos solo si y a menos que muestran un patrón semejante de respuestas. La dificultad de las inferencias NA en el caso de $a$ menos que se explicaría por el hecho de que el modelo negado es incompleto: "(no $q$ y) no $p$ ". Al recibir la premisa categórica "no $p$ ”, los razonadores se encontrarían con una dificultad ańadida, ya que tendrían que completar el modelo para llegar a la conclusión "no q". El que esta dificultad de NA se mantenga en el caso de solo si plantea la posibilidad de que este modelo negado sea también incompleto para este enunciado. 
No obstante, como ya encontraron Wright y Hull (1986, I988), los resultados muestran una diferencia entre estos dos enunciados; los enunciados a menos que son más difíciles que los enunciados solo si: el porcentaje global de inferencias en solo si fue significativamente más alto $(85 \%)$ y más rápido $(3.7 \mathrm{~s})$ que el de $a$ menos que ( $76 \%$ y $4.7 \mathrm{~s}$, respectivamente).

La semejanza propuesta por Fillenbaum para solo si y a menos que ha sido comprobada con material abstracto y arbitrario; queda por aclarar qué pasa cuando les pedimos que realicen las mismas inferencias condicionales con enunciados temáticos, con contenidos y contextos pragmáticos concretos.

\begin{tabular}{lcccc} 
& \multicolumn{4}{c}{ INFERENCIAS } \\
\cline { 2 - 5 } Condicionales & $\begin{array}{c}p, \\
\text { luego } q\end{array}$ & $\begin{array}{c}\text { no } p, \\
\text { luego no } q\end{array}$ & $\begin{array}{c}q, \\
\text { luego } p\end{array}$ & $\begin{array}{c}\text { no } q, \\
\text { luego no } p\end{array}$ \\
\hline "Si $p$ entonces $q "$ & MP: 98 & NA: 63 (5.0) & AC: 75 & MT: 78 (5.5) \\
& $(2.5)$ & & $(3.9)$ & \\
“ $p$ solo si $q "$ & MP: 94 & NA: 69 (4.7) & AC: 89 & MT: $90(3.9)$ \\
& $(3.3)$ & & $(2.9)$ & \\
"No $p$ a menos & MP: 76 & NA: $56(5.4)$ & AC: 86 & MT: $86(4.6)$ \\
que $q "$ & $(4.2)$ & & $(3.0)$ & \\
"Si no $q$ entonces & MT: 63 & AC: $64(5.3)$ & NA: 66 & MP: $96(3.4)$ \\
no $p "$ & $(5.6)$ & & $(4.6)$ &
\end{tabular}

Cuadro 4. Porcentajes de inferencias realizadas (y latencias en segundos) para los cuatro tipos de enunciados condicionales y los cuatro tipos de inferencias (MP, NA, AC y MT) del estudio de García-Madruga et al. 2008

\subsection{Las inferencias condicionales en "solo si" y "a menos que" con diversos contenidos}

En uno de los experimentos de Carriedo y colaboradores (Carriedo et al. I999; Carriedo et al. 2002) se pidió a I 50 estudiantes universitarios que participaran en una prueba colectiva de papel y lápiz, en la que tenían que escribir en un cuadernillo la conclusión a las cuatro inferencias condicionales. Si pensaban que no era posible extraer una conclusión necesariamente válida tenían que escribir "No hay conclusión”. En el cuadernillo los participantes recibían las instrucciones de la tarea, antes de recibir los problemas deductivos con las inferencias condicionales con varios contenidos. Los participantes fueron asignados aleatoriamente a dos condiciones o grupos, una por cada tipo de enunciado: solo si $(n=37)$ y a menos que $(n=37)$, de forma que en cada grupo los participantes realizaron las inferencias a partir de uno de los tipos de enunciados. Entre los contenidos utilizados en los 
enunciados estaba el mismo contenido abstracto del trabajo anterior y dos nuevos contenidos y contextos: las advertencias, como en Una persona puede observar un eclipse de sol solo si lleva gafas protectoras, o No se puede observar un eclipse de sol a menos que se lleven gafas protectoras; y las amenazas, como en Saldrás a jugar solo si te terminas la sopa, o No saldrás a jugar a menos que termines la sopa.

En cada condición, los participantes tenían que resolver 4 problemas inferenciales en cada una de las inferencias MP, NA, AC y MT, así como 4 en cada uno de los contenidos que eran presentados en forma aleatoria. Las dos diferencias fundamentales con respecto al trabajo anterior, por tanto, fueron que la tarea ahora era una prueba colectiva de papel y lápiz que no permitía registrar los tiempos, e implicaba construir mentalmente la conclusión y escribirla, no evaluar la conclusión dada.

En el cuadro 5 se muestran los resultados relativos a solo si y a menos que, en ese orden, en las diferentes inferencias y contenidos. Se puede observar que los resultados en el contenido arbitrario son muy semejantes a los obtenidos en el trabajo anterior. El patrón de respuestas es semejante para ambos tipos de enunciados, no hay diferencias entre MP y MT, en NA se produce un descenso claro en el porcentaje de inferencias, y el porcentaje global de inferencias es menor en a menos que que en solo si.

\begin{tabular}{lcccc} 
& \multicolumn{4}{c}{ InFERENCIA } \\
\cline { 2 - 5 } Contenido & $\begin{array}{c}\text { MP }(p, \\
\text { luego } q)\end{array}$ & $\begin{array}{c}\text { NA (no } p, \\
\text { luego no } q)\end{array}$ & $\begin{array}{c}\text { AC }(q, \\
\text { luego } p\end{array}$ & $\begin{array}{c}\text { MT (no } q, \\
\text { luego no } p \text { ) }\end{array}$ \\
\hline Arbitrario & $84-75$ & $37-44$ & $84-81$ & $89-72$ \\
Advertencias & $84-77$ & $27-58$ & $57-64$ & $84-81$ \\
Amenazas & $84-81$ & $73-89$ & $84-94$ & $89-94$ \\
\hline
\end{tabular}

Cuadro 5. Porcentajes de respuestas inferenciales

para los condicionales " $p$ solo si $q$ " y "no $p$ a menos que $q$ " en el experimento de Carriedo et al. 2002

En los contenidos temáticos de advertencias y amenazas, los resultados son diferentes que con el contenido abstracto. En las advertencias, tanto en solo si como en a menos que existe una clara disminución en las inferencias AC, mientras que las inferencias NA se mantienen bajas. Esto sugiere claramente que las advertencias facilitan la construcción del tercer modelo propio de la implicación material "no $p$ y $q$ " que bloquea las inferencias AC y NA. Esto se produce tanto en solo si como en a menos que, aunque con este último enunciado el porcentaje de inferencias NA sea 
significativamente más alto. Esto no resulta nada sorprendente, ya que en expresiones como Una persona puede observar un eclipse de sol solo si lleva gafas protectoras, o No se puede observar un eclipse de sol a menos que se lleven gafas protectoras, la posibilidad de llevar las gafas de protección sin que haya eclipse, es decir, por otro motivo o con otro propósito, es fácil de representar.

Los resultados inferenciales con las amenazas son diferentes de los dos contenidos anteriores. Tanto para solo si como para a menos que la interpretación que realizan los sujetos parece ser claramente bicondicional: no existen diferencias en el porcentaje de respuestas inferenciales para ninguna de las cuatro inferencias, ni tampoco existen diferencias entre los dos enunciados. De nuevo, los resultados son coherentes con el contexto pragmático de las amenazas, ya que, como vemos en nuestro ejemplo anterior, cuando una madre dice: Saldrás a jugar solo si te terminas la sopa, o No saldrás a jugar a menos que termines la sopa, está diciendo que el niño podrá salir a jugar si y solo si termina la sopa. La representación completa de ambos modelos iniciales es, por tanto, la representación final probable que construyen los participantes en el caso de las amenazas.

Como vemos, diferentes contenidos y contextos conducen a interpretaciones y representaciones diferentes de solo si y a menos que, aunque todas ellas confirman una doble representación inicial que confirma la ausencia de diferencias entre las inferencias MP y MT.

\subsection{La hipótesis de Dancygier: los condicionales "a menos que" y las disyunciones}

Como vimos, la representación que propone Barbara Dancygier (1998, 2002; también en Dancygier y Sweet 2005, Traugott 1997) para los condicionales "no $p$ a menos que $q$ " destaca su carácter exceptivo al ser interpretados en términos de dos enunciados: uno que afirma la probable negación de la proposición antecedente "no $p$ " y otro que afirma la única posibilidad de relación entre las proposiciones antecedente y consecuente: " $p$ y $q$ ". Dancygier y Sweeter (2005: I 87) sostienen que una característica del significado de a menos que es su inherente alternatividad, es decir, o se da "no $p$ " o se da " $p$ y $q$ ", lo que confirma la opción por la bicondicionalidad de su propuesta. La hipótesis de Dancygier, por tanto, coincide con la representación inicial que hemos propuesto anteriormente (véase el cuadro 3):

(no- $q$ y) no- $p$

$q$ у $p$

Por otra parte, la alternatividad de la representación de los enunciados a menos que se pone de manifiesto por su semejanza semántica con las expresiones disyuntivas. En español y en inglés, así como en otras muchas lenguas, la equivalencia lógi- 
ca entre condicionales y disyunciones con una proposición negada se pone de manifiesto en que este tipo de enunciados disyuntivos contienen un significado condicional (cf. Montolío 1999: 3697). Así, la representación de "o $q$ o no- $p$ " implica también dos posibilidades iniciales, ambas incompletas: o se da $q$ o se da no-p; según la teoría de modelos:

$$
q(\mathrm{y} p)
$$

(no- $q$ y) no- $p$

La semejanza semántica entre los enunciados a menos que y los condicionales disyuntivos queda reflejada en nuestros ejemplos anteriores de advertencias y amenazas que podrían ser formulados ahora como: $O$ se llevan las gafas protectoras o no se podrá observar un eclipse de sol, y $O$ te terminas la sopa o no saldrás a jugar.

En un estudio reciente (García-Madruga, Carriedo y Moreno-Ríos 20 I I) hemos comparado los condicionales a menos que con sus equivalentes disyuntivos, en una tarea de inferencias condicionales, manipulando al mismo tiempo la complejidad de la tarea. Para ello, hemos cambiado el orden de presentación de las premisas categóricas. De esta manera, la premisa categórica de cada problema deductivo podía ser presentada en el orden canónico, después de la premisa proposicional (por ejemplo, en una inferencia NA: "no $p$ a menos que $q$ ", "no- $p$ ", ¿Qué se puede concluir?) o en el orden de presentación inverso, antes de la premisa proposicional (por ejemplo, en una inferencia AC: “ $q$ ”, “o $q$ o no- $p$ ”, ¿Qué se puede concluir?). Como ya analizamos, el proceso de comprensión y razonamiento, de construcción y manipulación de las posibilidades o modelos se lleva a cabo dentro de la MO. Esta está caracterizada por su limitada capacidad de almacenamiento y procesamiento. Al presentar primero la premisa categórica, su comprensión produce una sobrecarga en la MO justo antes de que el individuo tenga que enfrentarse a la comprensión del enunciado, es decir, la construcción de las posibilidades que implica su significado. Por tanto, el orden de presentación inverso debería empeorar la actuación de los participantes y disminuir el porcentaje de respuestas inferenciales, especialmente en las inferencias que no pueden ser realizadas directamente debido a la representación incompleta, esto es, NA ("no $p$ ", luego "no- $q$ ") para $a$ menos que y NA y AC (“q", luego " $p$ ”) para las disyunciones. Las posibilidades tienen que ser completadas para realizar estas inferencias

Los participantes fueron i I 5 estudiantes universitarios que resolvieron cada uno I 6 problemas deductivos relativos a la relación entre personas y lugares en un juego que hacían los conductores de una empresa de transportes urgentes. Por ejemplo, Carlos no irá a Madrid a menos que Marta vaya a Sevilla, o su versión disyuntiva: $O$ Marta va a Sevilla o Carlos no irá a Madrid. Los participantes fueron asignados 
aleatoriamente en cuatro condiciones o grupos según los problemas que resolvieron, ya fueran a menos que o disyuntivos, y en el orden canónico (con la premisa categórica después del enunciado) o en el orden inverso (con la premisa categórica antes del enunciado).

\begin{tabular}{lcccc} 
& $\begin{array}{c}\mathrm{MP}(p, \\
\operatorname{luego} q)\end{array}$ & $\begin{array}{c}\mathrm{AC}(q, \\
\operatorname{luego} p)\end{array}$ & $\begin{array}{c}\mathrm{MT}(\text { no } q, \\
\text { luego no } p)\end{array}$ & $\begin{array}{c}\text { DA (no } p, \\
\text { luego no } q)\end{array}$ \\
\hline $\begin{array}{l}\text { Orden canónico } \\
\text { "No } p \text { a menos que } q "\end{array}$ & 86 & 89 & 82 & 84 \\
"O $q$ o no $p$ " & 59 & 49 & 54 & 53 \\
Orden inverso & & & & \\
"No $p$ a menos que $q "$ & 71 & 78 & 77 & $59^{*}$ \\
"O $q$ o no $p$ " & 52 & $39^{*}$ & 48 & $32^{*}$ \\
\hline
\end{tabular}

Cuadro 6. Porcentajes de respuestas inferenciales en el experimento de García-Madruga, Carriedo y Moreno-Ríos 20 I I (el asterisco [*] quiere decir que los porcentajes de respuestas que señala son significativamente menores que los que aparecen en la columna a su izquierda)

Los resultados de ambos enunciados en los dos órdenes pueden observarse en el cuadro 6. Un primer resultado muestra que los problemas con disyunciones fueron más difíciles que los problemas con a menos que, lo que revela la menor familiaridad de los participantes con los enunciados condicionales disyuntivos, al menos con contenidos como los utilizados en el experimento. En el orden canónico se confirma la semejanza en la representación de dos modelos para ambos enunciados: no hay diferencias significativas en los porcentajes de respuestas inferenciales ni para a menos que ni para las disyunciones. Como vemos, los resultados de a menos que son semejantes a los encontrados con las amenazas en el estudio presentados anteriormente. Por último, la introducción del cambio en el orden de presentación ha aumentado la dificultad de la tarea, ya que el porcentaje de respuestas inferenciales ha disminuido claramente en todos los casos. Además, el orden inverso ha permitido que aparezcan las diferencias entre las cuatro inferencias. En a menos que, la inferencia negativa NA resulta ahora significativamente más difícil que la otra inferencia negativa: MT; y en las disyunciones, además de que NA sea más difícil que MT, ahora la inferencia AC resulta también más difícil que MP. Los resultados, por tanto, confirman las representaciones propuestas para ambos tipos de enunciados y la hipótesis de Dancygier sobre el significado de a menos que.

Como vemos, nuestros resultados subrayan la naturaleza adaptativa y estratégica del razonamiento deductivo humano (Cf. García-Madruga et al. 2000). Una pe- 
queña modificación en la tarea, como es el cambio del orden de presentación del enunciado y la premisa categórica, puede afectar a cómo se resuelven los problemas. Cuando la premisa proposicional es presentada primero, dado que la tarea es relativamente simple y no hay restricciones temporales, los razonadores construyen los dos modelos completos y no hay diferencias en el porcentaje de inferencias. El cambio en el orden de presentación, con la premisa categórica primero, sobrecarga la MO de algunos sujetos al obligarles a construir la representación del enunciado después de la comprensión de la premisa categórica, es decir, con la representación de esta ocupando ya un espacio en la MO. De esta manera, es más probable que utilicen una representación incompleta y menos demandante del enunciado, lo que llevaría a una disminución en las inferencias dependientes de esa representación incompleta.

Los resultados de este trabajo pueden ser interpretados a la luz de otras perspectivas teóricas sobre el procesamiento lingüístico. Así, la perspectiva "good enough" (Ferreira, Ferraro y Bailey 2002; Ferreira y Patson 2007) sostiene que el sistema de comprensión del lenguaje no proporciona siempre una representación precisa y detallada de las expresiones lingüísticas, sino que las representaciones creadas son únicamente "suficientemente buenas" para llevar a cabo la tarea. En línea convergente, Sanford y Graesser (2006) sostienen que un concepto clave en la comprensión del lenguaje es el de 'underspecification', según el cual el análisis semántico preciso y completo del lenguaje raramente se lleva a cabo, ya que resulta muy costoso en términos de procesamiento. Estas perspectivas son plenamente convergentes con la que hemos propuesto y subrayan el papel que la MO tiene en la comprensión y el razonamiento lingüístico.

\section{A MODO DE CONCLUSIÓN}

Hemos visto que los seres humanos comprenden y razonan de forma semejante con los condicionales complejos afirmativos, " $p$ solo si $q$ ", y negativos, "no- $p$ a menos que $q$ ", pero de forma diferente a como lo hacen con los condicionales convencionales "si $p$ entonces $q$ " y "si no $q$ entonces no $p$ ". También hemos visto que los rasgos principales de la comprensión y razonamiento con los condicionales de uno y otro tipo pueden ser explicados por la teoría de los modelos mentales a partir de las posibilidades que representan los individuos. De esta manera, aunque los diversos condicionales posean el mismo significado lógico, es decir, las posibilidades finales sean las mismas, las representaciones, especialmente las iniciales, son diferentes, ya que pueden incluir una o dos posibilidades explícitas, pueden ser construidas de " $p$ a $q$ " o de " $q$ a $p$ ", y pueden ser completas o incompletas. 
Ahora bien, como hemos visto, nuestra teoría sostiene que, en todos los casos, las interpretaciones que realizan los individuos de los enunciados, las representaciones que construyen, dependen de las características de la tarea y de los recursos, conocimientos y creencias que poseen. El que se haga una interpretación bicondicional o condicional depende de la formulación condicional utilizada, pero también de la capacidad cognitiva y el compromiso motivacional del individuo y, especialmente, como ya apuntamos al presentar las ideas de Fillenbaum, del contenido y contexto de los enunciados.

Este trabajo nos ha permitido mostrar también que, si queremos estudiar y conocer el desarrollo, características y limitaciones del razonamiento humano, necesitamos utilizar los conocimientos que nos proporcionan no solo la lógica sino también, y de manera muy importante, la lingüística. Por otro lado, en sus estudios los psicólogos emplean métodos y técnicas experimentales que pueden ser aprovechados por la lingüística y que proporcionan nuevas evidencias al propósito común de averiguar las relaciones entre cognición, lenguaje y realidad. Asimismo, la psicología proporciona conceptos y perspectivas teóricas que pueden ser muy relevantes para el lingüista, entre las que queremos destacar tres que se ponen de manifiesto en nuestro trabajo. Los estudios presentados han sido realizados desde una perspectiva inequívocamente cognitiva que otorga un papel central a la representación a la hora de predecir y explicar el pensamiento y la conducta humana. En segundo lugar, estos estudios han sido realizados desde una concepción teórica específica, la teoría de los modelos mentales, que permite abordar de forma precisa y coherente los problemas de la cognición y que está teniendo una influencia relevante en diversos campos de la ciencia cognitiva. Por último, como se sostiene desde la teoría de modelos, en nuestro trabajo hemos utilizado ampliamente un concepto psicológico clave en la explicación del funcionamiento de la cognición: el concepto de memoria operativa (MO) o de trabajo (working memory). El estudio de la mente, la cognición y el lenguaje no puede ser abordado de forma reduccionista y aislada, encapsulada. Solo si las diversas perspectivas cognitivas se entrelazan y cooperan será posible llevar a cabo con éxito nuestra tarea como científicos cognitivos. Es más, la perspectiva proporcionada por las otras disciplinas es una fuente inagotable de ideas, reflexiones y evidencias que resultan especialmente productivas en cada uno de los campos específicos de la ciencia cognitiva.

\section{REFERENCIAS BIBLIOGRÁFICAS}

Barrouillet, Pierre, Nelly Grosset y Jean-François LeCAS (2000): "Conditional reasoning by mental models: Chronometric and developmental evidence”, Cognition, 75, pp. 237-266. https://doi.org/I0.1016/soo I0-0277(00)00066-4. 
Braine, Martin D. S. y David O’Brien (eds.) (1998): Mental Logic, Mahwah (NJ): Lawrence Erlbaum Associates.

Byrne, Ruth M. J. (2005): The Rational Imagination. How People Create Alternatives to Reality, Cambridge (MA): MIT Press. https://doi.org/I0.755 I/mitpress/5756.00I.000I.

ByRne, Ruth M. J. y Suzanne M. EGAN (2004). "Counterfactual and prefactual conditionals", Canadian Journal of Experimental Psychology, 58, pp. I I 3-г 20. https://doi.org/I0.1037/hoo8579I.

ByRne, Ruth M. J. y Alessandra TASSO (1999): "Deductive reasoning with factual, possible and counterfactual conditionals”, Memory and Cognition, 27, pp. 726-740. https://doi.org/I0.3758/bfo32 I I 565 .

Carriedo, Nuria, Juan A. García-Madruga, Francisco Gutiérrez y Sergio Moreno-Ríos (I999): "How does content affect 'unless' conditional reasoning?”, en Sebastiano Bagnara (ed.), Proceedings of the European Conference on Cognitive Science 1999, Rome: Istituto di Psicologia / Instituto di Psicologia Consiglio Nazionale delle Recerche, pp. 27I-277.

Carriedo, Nuria, Juan A. García-Madruga, Sergio Moreno-Ríos y Francisco Gutiérrez (2002): "The effect of content and formulation on conditional reasoning: The case of warnings and threats" (manuscrito no publicado).

DANCYGIER, Barbara (1998): Conditionals and Prediction. Time, Knowledge and Causation in Conditional Constructions, Cambridge: Cambridge University Press. https://doi.org/Io. Io I 7 /cbo97805 I I 486463.

DANCYGIER, Barbara (2002): "Mental space embeddings, counterfactuality and the use of unless", English Language and Linguistics, 6, pp. 347-377. https://doi.org/10. I017/sI 360674302000278.

DANCYGIER, Barbara y Eve SwEETSER (2005): Mental Spaces in Grammar. Conditional Constructions, Cambridge: Cambridge University Press. https://doi.org/Io. I I 77/0075424209354957.

Delval, Juan A. y Mario CARRetero (1978): "La adquisición de las conectivas proposicionales", en Juan Delval (comp.), Lecturas de psicología del niño, Madrid: Alianza, vol. 2, pp. I68-i94.

EgAN, Suzanne M., Juan A. GARCÍA-MADrUGA y Ruth M, J. BYRnE (2009): "Indicative and Counterfactual 'only if conditionals", Acta Psychologica, I33, pp. 240-249. https://doi.org/I0.1016/j.actpsy.2009.07.005.

Evans Jonathan St B. T. (I 977): "Linguistic factors in reasoning”, Quaterly Journal of Experimental Psychology, 29, pp. 197-206.

Evans Jonathan St B. T., David E. Over y Simon J. HANDley (2005): "Supposition, extensionality and conditionals: A critique of Johnson-Laird y Byrne (2002)”, Psychological Review, I I 2(4), pp. I040-I052. https://doi.org/I0.1037/0033-295X. I I 2.4.1040.

Ferreira, Fernanda, Vittoria Ferraro y Karl G. D. BAILEY (2002): "Good-enough representations in language comprehension”, Current Directions in Psychological Science, I I, pp. I I-I 5. https://doi.org/I0.I I I I/I 467-872 I.00I 58 .

Ferreira, Fernanda y Nikole D. PATSON (2007): The 'Good Enough' approach to language comprehension, Language and Linguistics Compass, I ( I-2), pp. $7 \mathrm{I}-83$.

https://doi.org/I0.I I I I/j.I 749-8 I 8x.2007.00007.x.

FILlENBAUM, Samuel (I 976): "Inducements: On phrasing and logic of conditional promises, threats and warnings", Psychological Research, 38, pp. 23 I-20. https://doi.org/10.1007/bfoo309774. 
FILLENBAUM, Samuel (1986): "The use of conditionals in inducements and deterrents", en Elisabeth ClossTraugott, Alice Ter Meulen, Judy Snitzer Reilly y Charles A. Ferguson (eds), On conditionals, Cambridge: Cambridge University Press, pp. I79-195.

https://doi.org/I o. IOI 7/cbo97805 I I753466.o Io.

García-Madruga, Juan A., Sergio Moreno, Nuria CARriedo y Francisco Gutiérrez (2000): "Task, premise order and strategies in Rips's conjunction, disjunction and conditionals problems”, en Walter Schaeken, Gino De Vooght, André Vandierendonk y Géry D’Ydewalle (eds.), Deductive Reasoning and Strategies, Mahwah (NJ): Lawrence Erlbaum Associates, pp. 49-72.

García-Madruga, Juan A., Sergio Moreno, Nuria Carriedo, Francisco Gutiérrez y Philip N. JOHNSON-LAIRD (200I): "Are conjunctive inferences easier than disjunctive inferences? A comparison of rules and models", The Quaterly Journal of Experimental Psychology, 54A(2), pp. 6I 3-632. https://doi.org/IO.1080/713755974.

García-Madruga, Juan A., Nuria Carriedo, Francisco Gutiérrez, Sergio Moreno y Philip N. JOHNSON-LAIRD (2002): "Mental models in deductive reasoning", Spanish Journal of Psychology, 5(2), PP. I25-I 40.

García-Madruga, Juan A., Nuria Carriedo, Sergio Moreno, Francisco Gutiérrez y Walter SCHAEKEN (2008): “Unless Reasoning”, Spanish Journal of Psychology, I I (2), pp. 386-399.

García-Madruga, Juan A., Sergio Moreno-Ríos, Ana Cristina QuelHas y Csongor Juhos (2009): "Reasoning with 'Unless' counterfactual conditionals", Psicologica, 30, pp. 2 1 5-242.

García-Madruga, Juan A., Nuria Carriedo y Sergio Moreno-Ríos (20 i i): "The semantics of 'unless' conditionals: Evidence from 'unless' and disjunctive inferences", Spanish Journal of Psychology, I4(2), pp. 569-579. https://doi.org/I0.5209/rev_sjop.20 I I.vi 4.n2.5.

García Madruga, Juan A., Carlos Santamaría y Sergio Moreno Ríos (2016): "Pensamiento y Razonamiento", en Teresa Bajo Molina, Luis José Fuentes Melero, Juan Lupiánez Castillo, Charo Rueda Cuerva (coords.), Mente y cerebro. De la psicología experimental a la neurociencia cognitiva, Madrid: Alianza, cap. I4, pp. 419-442.

GEIS, Michael L. (I973): "If and unless", en Braj B. Kachru, Robert B. Lees, Yakov Malkiel, Angelina Pietrangeli y Sol Saporta (eds), Issues in Linguistics. Papers in Honor of Henry and Renée Kahane, Urbana-Champaign (IL): University of Illinois Press, pp. 23 I-253. https://doi.org/I0.1525/aa.1976.78.3.02a00800.

InHELDER, Bärbel y Jean PIAGET (1972 [1955]) : De la lógica del niño a la lógica del adolescente (trad. María Teresa Cevasco), Buenos Aires: Paidós.

JefFreY, Richard (1981): Formal Logic. Its Scope and Limits, New York: McGraw-Hill. https://doi.org/I0.2307/2271990.

JohnsOn-LaIRD, Philip N. (I983): Mental models. Toward a Theory of Inference, Language and Consciousness, Cambridge (MA): Harvard University Press.

JOHnSOn-LaIRD, Philip N. (I999): "Deductive reasoning”, Annual Review, 50, pp. I09-I35.

JoHnSON-LAIRD, Philip N. (2006): How We Reason. Oxford (UK): Oxford University Press.

JOHNSON-LAIRD, Philip N. y Ruth M. J. BYRNE (I989): “Only reasoning”, Journal of Memory and Language, 28(3), pp. 313-330. https://doi.org/го. I I6/0749-596x(89)90036-3.

Johnson-Laird, Philip N. y Ruth M. J. BYRne (I99I) : Deduction, Hove (UK): Erlbaum. 
Johnson-Laird, Philip N. y Ruth M. J. BYRNE (2002): "Conditionals: A theory of meaning, pragmatics, and inference", Psychological Review, ı09(4), pp. 646-678.

https://doi.org/I0.1037//0033-295x.109.4.646.

JOHnSON-LAIRD, Philip N., Ruth M. J. BYRnE y Walter SCHAEKEN (I992): "Propositional reasoning by model", Psychological Review, 99(3), pp. 4I 8-439.

https://doi.org/I0.1037//0033-295x.99-3.4I 8.

MONTOLÍO, Estrella (I999): "Las construcciones condicionales”, en Ignacio Bosque y Violeta Demonte (dirs.), Gramática descriptiva de la lengua española, Madrid: Espasa Calpe, vol. 3, pp. 3643-3738.

MORENO-RÍOS, Sergio y Juan A. GARCÍA-MADRUGA (2002): "El desarrollo del razonamiento sobre lo que podría haber ocurrido: condicionales indicativos y subjuntivos", Infancia y Aprendizaje, 25(4), pp. 485-498. https://doi.org/I0. I I 74/021037002762064055.

Oberauer, Klaus y Oliver WilHelm (2003): "The meaning(s) of conditionals: Conditional probabilities, mental models and personal utilities", Journal of Experimental Psychology: Learning, Memory, and Cognition, 29(4), pp. 680-693. https://doi.org/10.1037/0278-7393.29.4.680.

QUINE, W. V. (1972): Methods of Logic, New York: Holt, Rinehart y Winston.

ReICHENBACK, Hans (1 947): Elements of Symbolic Logic, New York: Free Press.

RIPS, Lance J. (1994): The Psychology of Proof. Deductive Reasoning in Human Thinking, Cambridge (MA): MIT Press.

SANFORD, Anthony J. y Arthur C. Graesser (2006): "Shallow processing and underspecification", Discourse Processes, 42(2), pp. 99-I08. https://doi.org/IO.I207/sI 5326950dp4202_I.

TAPLIN, John E., Herman STAUdENMAYER y Judith L. TADDONIO (I974): "Developmental changes in conditional reasoning: Linguistic or logical?", Journal of Experimental Child Psychology, I7(2), pp. 360-73. https://doi.org/I0.1016/0022-0965(74)90080-0.

TraugOTT, Elisabeth Closs (1997): "Unless and but conditionals: A historical perspective", en Angeliki Athanasiadou y René Dirven (eds.), On Conditionals Again, Amsterdam: John Benjamins. https://doi.org/I0.1075/cilt. I 43.09clo.

WRIGHT, Patricia y Audrey J. Hull (1986): “Answering questions about negative conditionals”, Journal of Memory and Language, 25(6), pp. 69i-709. https://doi.org/Io.1016/0749-596X(86) 90044-6.

Wright, Patricia y Audrey J. Hull (1988): "Reading to do: Creating contingent action plans", British Journal of Psychology, 79(2), pp. I87-2 I I. https://doi.org/Io.I I I I/j.2044-8295.I 988. tbo2283.x. 